\title{
A Lightweight Hyperspectral Mapping System and Photogrammetric Processing Chain for Unmanned Aerial Vehicles
}

\section{Juha Suomalainen ${ }^{1, *}$, Niels Anders ${ }^{2}$, Shahzad Iqbal ${ }^{3}$, Gerbert Roerink ${ }^{4}$, Jappe Franke ${ }^{4}$,} Philip Wenting ${ }^{1}$, Dirk Hünniger ${ }^{3}$, Harm Bartholomeus ${ }^{1}$, Rolf Becker ${ }^{3}$ and Lammert Kooistra ${ }^{1}$

1 Laboratory of Geo-Information Science and Remote Sensing, Wageningen University, Wageningen 6708PB , The Netherlands; E-Mails: philip.wenting@wur.nl (P.W.); harm.bartholomeus@wur.nl (H.B.); lammert.kooistra@wur.nl (L.K.)

2 Soil Physics and Land Management Group, Wageningen University, Wageningen 6708PB, The Netherlands; E-Mail: niels.anders@wur.nl

3 Hochschule Rhein-Waal, 47533 Kleve , Germany; E-Mails: shahzad.iqbal@gmail.com (S.I.); Dirk.Huenniger@hochschule-rhein-waal.de (D.H.); Rolf.Becker@hochschule-rhein-waal.de (R.B.)

4 Alterra, Wageningen 6708PB, The Netherlands; E-Mails: gerbert.roerink@wur.nl (G.R.); jappe.franke@wur.nl (J.F.)

* Author to whom correspondence should be addressed; E-Mail: juha.suomalainen@wur.nl; Tel.: +31-6-3732-3178.

External Editors: Arko Lucieer, Gonzalo Pajares Martinsanz and Prasad S. Thenkabail

Received: 3 June 2014; in revised form: 20 October 2014 / Accepted: 3 November 2014 / Published: 10 November 2014

\footnotetext{
Abstract: During the last years commercial hyperspectral imaging sensors have been miniaturized and their performance has been demonstrated on Unmanned Aerial Vehicles (UAV). However currently the commercial hyperspectral systems still require minimum payload capacity of approximately $3 \mathrm{~kg}$, forcing usage of rather large UAVs. In this article we present a lightweight hyperspectral mapping system (HYMSY) for rotor-based UAVs, the novel processing chain for the system, and its potential for agricultural mapping and monitoring applications. The HYMSY consists of a custom-made pushbroom spectrometer (400-950 nm, 9 nm FWHM, 25 lines/s, 328 px/line), a photogrammetric camera, and a miniature GPS-Inertial Navigation System. The weight of HYMSY in ready-to-fly configuration is only $2.0 \mathrm{~kg}$ and it has been constructed mostly from off-the-shelf components. The processing chain uses a photogrammetric algorithm to produce a Digital
} 
Surface Model (DSM) and provides high accuracy orientation of the system over the DSM. The pushbroom data is georectified by projecting it onto the DSM with the support of photogrammetric orientations and the GPS-INS data. Since an up-to-date DSM is produced internally, no external data are required and the processing chain is capable to georectify pushbroom data fully automatically. The system has been adopted for several experimental flights related to agricultural and habitat monitoring applications. For a typical flight, an area of 2-10 ha was mapped, producing a RGB orthomosaic at $1-5 \mathrm{~cm}$ resolution, a DSM at 5-10 cm resolution, and a hyperspectral datacube at $10-50 \mathrm{~cm}$ resolution.

Keywords: Unmanned Aerial Vehicle (UAV); hyperspectral mapping system; agriculture; remote sensing; photogrammetry

\section{Introduction}

Over the past decades, hyperspectral imaging or imaging spectroscopy has been established as a remote sensing technology that allows quantitative characterization of the earth system [1]. More specifically, it allows the identification of surface materials, the measurement of (relative) concentrations and the assignment of proportions of surface components within mixed pixels. For example, for applications related to vegetation science, earlier studies have shown that an increased spectral resolution available from hyperspectral systems provide the opportunity to estimate vegetation properties like chlorophyll, nitrogen [2] and leaf water content [3] with improved accuracies.

Although hyperspectral imaging is recognized as a promising technique, the number of operational sensors mounted on either manned full size aircrafts or satellites is still rather limited when compared to available multispectral sensor systems. Practical issues (non-regular data acquisition, lack of flexibility and high costs) have so far resulted in limited practical use of hyperspectral remote sensing data in the above-mentioned domains. However, during the last decade the new remote sensing platform of Unmanned Aerial Vehicles (UAVs) has come into wide use, allowing more widespread experimentation and application of hyperspectral imaging [4]. UAVs are (semi-) autonomously flying aircraft systems, ranging from helicopters of a few centimeters to full-size airplanes [5]. Depending on the context, UAVs are often referred also as Remotely Piloted Aircraft Systems (RPAS) or Unmanned Aerial Systems (UAS). For remote sensing research use the most interesting class of UAVs consist of those that are large enough to carry camera systems but still small enough to flexibly operate outside official airports. Typical UAVs of this class are multicopters and small airplanes capable of carrying camera payload approximately between $300 \mathrm{~g}$ and $10 \mathrm{~kg}$.

Such small UAVs can easily be equipped with consumer cameras and are used to take aerial photos for both research and commercial purposes [4]. Common processing techniques for these aerial image datasets include the use of ready photogrammetric software packages in generation of orthomosaics [6], 3D point clouds, and Digital Surface Models (DSM) [7].

Currently the most lightweight commercially available hyperspectral pushbroom systems weigh more than $3 \mathrm{~kg}$, including all mandatory equipment, such as a data logger, a mounting frame, and batteries. Several research groups [8-11] have already successfully mounted and operated hyperspectral 
pushbroom sensors of that weight class on UAVs. Although the UAVs capable of carrying 3-10 kg payloads are a feasible option in many cases, there are certain benefits in downscaling the technology for even smaller UAVs. The smaller UAVs are generally cheaper, safer, and easier to operate from both a physical and regulatory point of view. Furthermore, even if larger UAVs are used for other reasons, such as increased wind stability, a lower sensor weight allows mounting of larger flight batteries making longer flights possible.

Pushbroom data are commonly georeferenced using a processing method called direct georeferencing [12] where the scan lines are projected onto a Digital Surface Model (DSM) or a flat ground based solely on external orientation data (X, Y, Z, Roll, Pitch ,Yaw). This orientation data is acquired with a GPS-Inertial Navigation System (GPS-INS) synchronized to the pushbroom sensor exposures. To achieve operational georeferencing accuracy, the GPS-INS accuracy must at least be in the order of the sensor pixel. This usually requires a RTK-GPS instrument with $\mathrm{cm}$ accuracy and a heavy gyroscopic inertial measurement unit. Because of such high requirements, the GPS-INS units are often the heaviest and most expensive components in the current UAV pushbroom systems.

Another requirement for direct georeferencing is the availability of a DSM. For the processing of airplane and satellite pushbroom data, generic elevation models are used but their drawback is that they usually don't include the elevation of vegetation and man-made objects. Given the typical 1-10 m resolution of airplane data, this is not a critical flaw, but with the potential centimeter resolution that can be achieved with UAV acquired data, the availability of a more detailed DSM is essential. Currently, most pushbroom UAV operators work around the non-availability of a DSM by first projecting their data over a generic elevation model and then by manually rubber-sheeting the data over a previous acquired image of the area.

In this paper, we first present the UAV based Hyperspectral Mapping System (HYMSY) developed at Wageningen University and Research Centre. HYMSY is a lightweight $(2.0 \mathrm{~kg})$ hyperspectral pushbroom system developed for small UAVs. HYMSY consists of a custom-made pushbroom spectrometer, a small but inaccurate GPS-INS system, and a consumer camera. HYMSY is capable of producing in one dataset a georeferenced hyperspectral datacube, an aerial image orthomosaic, and a detailed 3D model of the target area. Secondly, we present a novel automated photogrammetric processing chain for accurate georectification of the HYMSY data. The photogrammetric processing chain solves both the lack of high quality GPS-INS data and the need for a detailed DSM. By collecting aerial images simultaneously with the pushbroom data, it is possible to perform accurate and fully automated georeferencing without the need for a high quality GPS-INS system or any external DSM. Finally, we demonstrate a case study dataset collected using HYMSY and briefly evaluate its performance in an agricultural mapping application.

\section{Hyperspectral Mapping System (HYMSY)}

\subsection{System Design}

In autumn 2012, our research team decided to acquire an UAV based hyperspectral mapping system. In order to do agricultural research on single plant basis, we needed the system to create hyperspectral reflectance factor maps at a spatial resolution of at least $10 \mathrm{~cm}$ resolution and with a 
georeferencing accuracy of a few pixels. The spectral resolution of $10 \mathrm{~nm}$ was estimated well enough for advanced red-edge analysis while still providing enough light for short exposure times. We selected an octocopter as the UAV platform because it allows flexible flight plans suitable for controlled research use. As commercial solutions that could be fitted in a small UAV multicopter with a maximum payload capacity of $2.0 \mathrm{~kg}$ were not available, we decided to develop our own design. Ideally the spectrometer should have covered also the SWIR range, but limitations regarding the minimal dimension available for this camera technology forced us to stick with 400-950 nm range available as CMOS sensor.

Our desktop study on commercial pushbroom spectrometers revealed that at that the commercial solutions available were not really optimized for use on a small UAV but were rather directly downscaled versions of earlier manned airplane size systems. The miniaturization to $2.0 \mathrm{~kg}$ threshold was found to be possible by simply selecting the smallest off-the-shelf components. As an innovation to hyperspectral data processing, we decided to include a photogrammetric camera to the system, which would allow automated production of the DSM for the target area and enable photogrammetric orientation of the system.

The HYMSY consists of a custom pushbroom spectrometer, a consumer camera, a compact GPS-INS unit, a RaspBerry PI data sink, custom synchronization electronics, and a lithium polymer battery (3S, 500-1000 mA). Figure 1 and Table 1 present the technical details of the system. We designed the HYMSY to be mounted under an octocopter UAV (Aerialtronics Altura AT8 v1, Figure 1), but it is a standalone setup that can be installed on any platform able to carry a $2.0 \mathrm{~kg}$ payload. The total price of components is approximately $12,000 €$.

Figure 1. Schematic figure of the HYMSY frame and main components (Left) Photo of HYMSY mounted on an Aerialtronics Altura AT8 v1 octocopter UAV (Right).
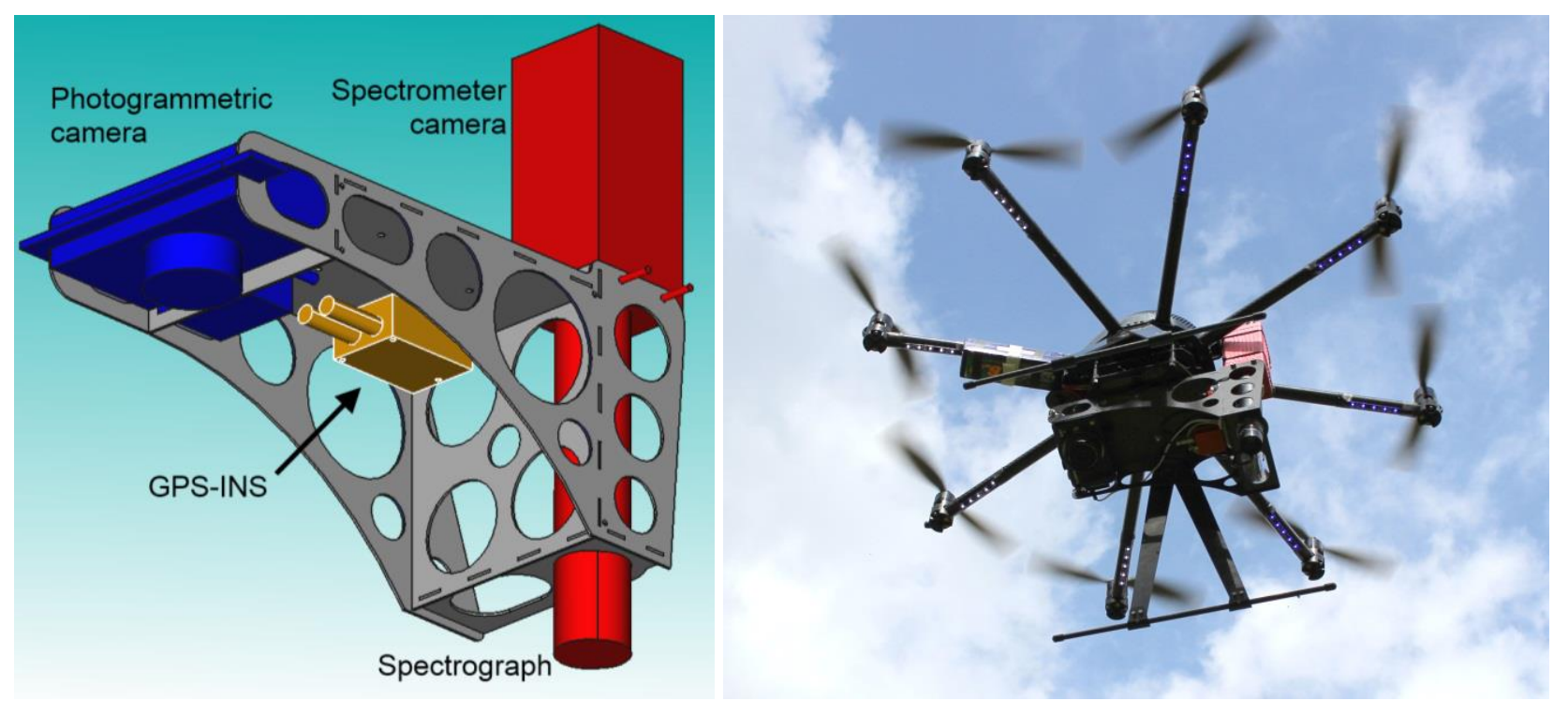
frames and GPS-INS messages are sent over an Ethernet connection to the RaspBerry PI data sink where they are stored to a USB flash drive.

The consumer camera photos are not extracted from the camera in real time, but are recorded to the SD memory card inside the camera. This produces a challenge to reliable pairing between the trigger pulses and photo files. To do this reliably the camera is started with a special start-up sequence where the first three photos are shot in very quick succession after which a pause of exactly $10 \mathrm{~s}$ is held before starting the normal acquisition at $2 \mathrm{~s}$ intervals. The Panasonic RW2 raw files contain the photo storage times with $10 \mathrm{~ms}$ accuracy, which allows the identification of the start-up sequence images and detection of possible missed frames.

Figure 2. Schematic figure of the HYMSY synchronization pulses and the data flow. The DSP outputs from I/O-1 a 1-ms pulse on every spectrometer exposure $(25 \mathrm{~Hz})$ to trigger the GPS-INS. Every $2 \mathrm{~s}$ the DSP triggers the photogrammetric camera (Panasonic GX1) with a $83-\mathrm{ms}$ wide pulse from $\mathrm{I} / \mathrm{O}-2$. The time lag of $83 \mathrm{~ms}$ is the delay between the trigger rising edge and the camera exposure, allowing use of the falling edge of the trigger pulse to synchronize GPS-INS to the camera exposure.

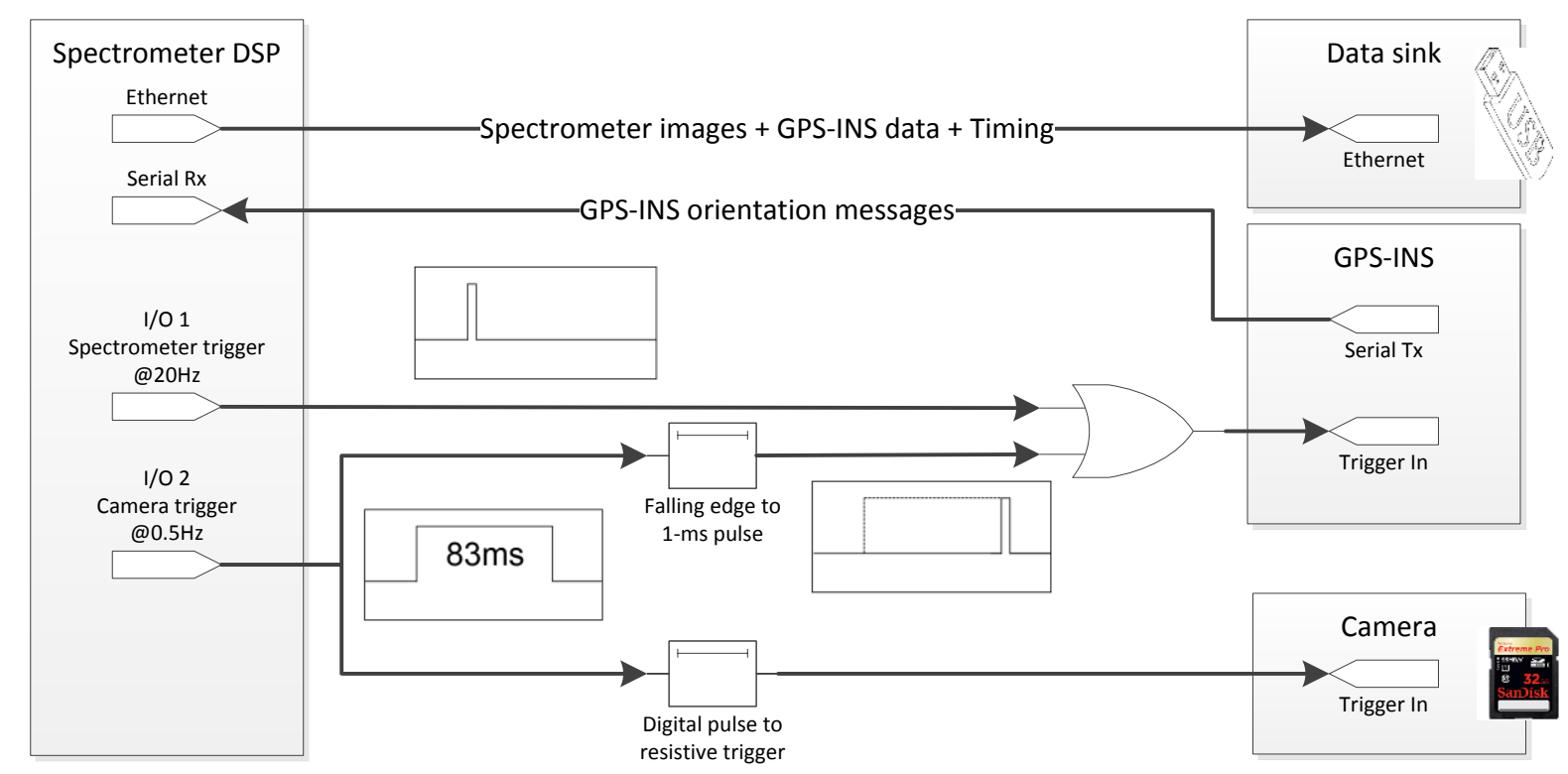

\subsection{Laboratory Calibrations}

The spectral calibration was performed by taking spectrometer images of a white panel illuminated with a spectral emission lamp. A few hundred of these images were averaged to a single low noise image. The emission line positions were determined separately for each spatial column of the image and a second order polynomial was fitted to them to form a pixel-wise spectral calibration. This simple calibration method provides only the central wavelength calibration, but does not take in to account more delicate issues, such as thermal effects, spectral resolution, stray light, or instrument line shape.

The pixel-wise dark current calibration of spectrometer was performed by taking a series of spectrometer images with the lens cap on. To increase the SNR the images were averaged together to form a pixel-wise dark current image. 
The flat field calibration of the spectrometer was performed by taking a series of images of a large integrating sphere illuminated with a Quartz-Tungsten-Halogen lamp. During the calibration measurements the spectrometer was constantly turned to randomize the view positions of each pixel. The spectrometer images were averaged and the dark current image was extracted from it to form the flat field image. Currently the sensor is assumed to have perfect linearity and thermal stability. If an absolute radiometric calibration was needed, then we also should have measured the radiance of the flat field. Currently, HYMSY is used only for relative reflectance factor measurements and thus flat field radiance was not recorded.

The synchronization timing calibrations include a series of measurements. The clock of the DSP is used as synchronization reference for all timings in the system. The exposure of the spectrometer camera is natively synchronized to the clock with better that $1 \mathrm{~ms}$ accuracy. With each spectrometer exposure the GPS-INS is triggered with a timed trigger pulse. Based on the specifications of the GPS-INS this synchronization is done with $2.5 \mathrm{~ms}$ accuracy. Although GPS-INS triggering is practically instantaneous, the output message is received with approximately $120 \mathrm{~ms}$ delay.

The Panasonic GX1 camera exposures are synchronized to the DSP clock by timing of the trigger pulses. The Panasonic camera has a delay of $83 \mathrm{~ms}$ between the rising edge of the trigger pulse and camera exposure. This delay was determined by splitting the trigger signal to a LED and placing it in the field of view of the camera. Then different trigger pulse widths were tested until LED was seen lighted up on half of the photos. As the falling edge of the pulse is used to trigger the GPS-INS, this calibration makes it certain that the Panasonic GX1 and the GPS-INS are synchronized.

The geometric calibration of the photogrammetric camera was performed using the Agisoft Lens (v0.4.1, Agisoft) calibration software. The boresight calibration parameters include the shift and rotation ( $\mathrm{dX}, \mathrm{dY}, \mathrm{dZ}, \mathrm{rX}, \mathrm{rY}, \mathrm{rZ}$ ) of the spectrometer, the photogrammetric camera, and the GPS-INS relative to the HYMSY frame. In the first stage, the shifts are measured with a ruler from the center point of the frame. For the spectrometer and the camera, the reference point is in the estimated focal point of the optics and for the GPS-INS it is in the GPS antenna. The rotations are determined iteratively. First the rotations are set coarsely allowing georectification of an airborne dataset to be performed adequately. Next the GPS-INS and photogrammetric camera rotations are adjusted so that separation between the photogrammetric alignments from PhotoScan and the GPS-INS data are minimized. Finally, the spectrometer rotations and spectrometer optics opening angle are adjusted so that the georectified spectrometer image overlaps the photogrammetric orthomosaic.

\subsection{Field Operation}

During an UAV mapping flight the following measurement procedure is performed. First, only the GPS-INS system is powered up for a minimum of 13 minutes before takeoff in order to achieve the highest positioning accuracy. 13 minutes is the time that a GPS satellite requires to transmit the full GPS almanac data. While gaining GPS data, the photogrammetric camera (Panasonic GX1) is turned on and configured. To ensure reliable and static performance, the camera is always used in the fully manual M-mode with all automation off. The camera exposure time is adjusted to match light conditions and lens focus is locked to infinity. 
To start the optical data acquisition also the rest of the HYMSY is powered on. After the start up, HYMSY enters the measurement cycle in which it collects 25 spectrometer scan lines per seconds and a photogrammetric image every two seconds. To calibrate the system for up-to-date dark current level, the first camera and spectrometer frames are taken with the lens caps on. To calibrate for the current illumination conditions, the caps are removed and the HYMSY is manually scanned over a Spectralon (Labsphere Inc) reference panel to acquire reference spectra.

After the calibration phase, the HYMSY is ready for the mapping flight. The mapping flight path is designed based on the required resolution which then defines the maximum flight speed and altitude. The flight path is uploaded to the UAV and an automated flight is performed. Once the UAV finishes the flight, a post flight Spectralon calibration is performed for HYMSY. To finish the measurement procedure, the HYMSY is powered down and data are extracted from the USB flash drive and the SD card to a field computer. The extracted dataset consists of a HYMSY timing txt-file, GPS-INS data bin-file, spectrometer frames as pgm-files $(1500 /$ minute $\times 65 \mathrm{kB}=97.5 \mathrm{MB} /$ minute $)$, and aerial images as rw2-files (30/minute $\times 19 \mathrm{MB}=570 \mathrm{MB} /$ minute $)$.

\section{Processing Chain}

The processing chain is based on Matlab (v2012b, Mathworks) scripts and interfaces. We have developed a simple user interface that allows selection of the source files, the destination folders, and setting the processing parameters. The actual processing is fully automated as once the parameters are set, the script runs also the external subprocesses in PhotoScan and PARGE to produce georeferenced outputs without any mandatory manual georeferencing steps. The processing chain can be separated to two successive phases where first all data is processed radiometrically and after that geometrically.

\subsection{Radiometric Processing}

As the first step of radiometric processing, the spectrometer images are converted from digital number $(D N)$ to radiance $(L)$ units using a pixelwise $(i j)$ dark current and flat field calibration according to the following equation:

$$
L^{i j}=\frac{D N^{i j}-D N_{\text {DarkCurrent }}^{i j}}{D N_{\text {FlatField }}^{i j}-D N_{\text {DarkCurrent }}^{i j}} L_{\text {FlatField }}\left(\lambda_{i j}\right)
$$

where $D N^{i j}$ is the intensity of a pixel in the spectrometer image at cross-track index $i$ and spectral dimension index $j, D N_{\text {DarkCurrent }}^{i j}$ is the intensity of the same pixel in the dark current calibration image, $D N_{\text {FlatField }}^{i j}$ is the pixel intensity in the flat field calibration image, and $L_{\text {FlatField }}\left(\lambda_{i j}\right)$ is the flat field radiance at the central wavelength of the pixel. As until now all the HYMSY data is field calibrated using a reflectance reference method and the end products are output only in reflectance factor units, performing an absolute radiometric calibration is not necessary. Therefore the flat field radiance was set to $\left(L_{\text {FlatField }}=1\right)$ thus making the output radiances to pseudo-radiance units.

Next, the raw wavelengths of the radiance images are converted to standardized output wavelengths. This allows that the spectra from different parts of the field of view can be compared directly. In the raw spectrometer image the spectral sampling is approximately every $3 \mathrm{~nm}$ but the raw wavelengths vary slightly for every cross-track column (spectral smile). To standardize the 
wavelengths, the image is resampled to $5 \mathrm{~nm}$ steps in 400-950 nm range. As the spectral resolution of the spectrograph is only $9 \mathrm{~nm}$, no significant data is lost in this process. The conversion is done by applying a 5-nm Gaussian filter to each column of the image effectively converting the original $328 \times 256$ image to $328 \times 111$ size using equation:

$$
L^{i j^{\prime}}=\frac{\sum_{j} \exp \left(-4 \ln (2) \frac{\left(\lambda_{i j}-\lambda_{j^{\prime}}\right)^{2}}{F W H M^{2}}\right) L^{i j}}{\sum_{j} \exp \left(-4 \ln (2) \frac{\left(\lambda_{i j}-\lambda_{j^{\prime}}\right)^{2}}{F W H M^{2}}\right)}
$$

where $\lambda_{i j}$ is the central wavelength of the pixel as defined in spectral calibration, $\lambda_{j^{\prime}}$ is the output wavelength, FWHM is the full width at half maximum of the filter, and $L^{i j^{\prime}}$ is the radiance at cross-track column $i$ in wavelength $\lambda_{j^{\prime}}$.

A typical HYMSY mapping flight with the octocopter UAV lasts 5-10 minutes and data is taken at maximum altitude of $120 \mathrm{~m}$ (because of legal restrictions). The short time span of the measurements and the low amount of atmosphere between the sensor and ground surface makes it is possible to convert the HYMSY spectrometer data to reflectance factors using the reflectance reference panel method. Within our mapping procedure, a 25\% Spectralon reference panel is measured with the HYMSY immediately before takeoff and after landing. Such calibration procedure allows correction for linear changes in the incident irradiance, which is beneficial in clear sky, stratus, and cirrus cloud weather situations [13].

To convert the radiances to reflectance factors, the reference panel pixels are manually selected from the unrectified hyperspectral datacube. The measured reference panel radiance spectra are averaged separately for the before and after flight panel measurements. The reflectance factor conversion is then applied using a linear interpolation of the reference panel radiances:

$$
R^{i j^{\prime}}=\frac{L^{i j^{\prime}}}{\frac{\left(t_{R e f_{2}}-t\right)}{\left(t_{R e f_{2}}-t_{R e f_{1}}\right)} L_{R e f_{1}}^{j^{\prime}}+\frac{\left(t-t_{R e f_{1}}\right)}{\left(t_{R e f_{2}}-t_{R e f_{1}}\right)} L_{R e f_{2}}^{j^{\prime}}} R_{R e f}\left(\lambda_{j^{\prime}}\right)
$$

where $R_{R e f}$ is the reflectance factor of the reference panel at $\lambda_{j^{\prime}}$ wavelength, $L_{R e f_{1}}^{j^{\prime}}$ and $L_{R e f_{2}}^{j^{\prime}}$ are the radiances measured of the reference panel before and after the flight, $t, t_{R e f_{1}}$, and $t_{R e f_{2}}$ are the exposure times of the spectrometer images and $R^{i j^{\prime}}$ is the final reflectance factor value for cross track pixel $i$ and output wavelength index $j^{\prime}$. Finally the reflectance factor images are merged to an unrectified datacube file in 16 bit ENVI BSQ format.

Optionally, using equations 1 and 3 it is also possible to do the reflectance factor calibration for the aerial images collected with the Panasonic GX1 camera. This allows production of high-resolution RGB reflectance factor imagery. However this is not a mandatory step for the hyperspectral processing.

\subsection{Geometric Processing}

The first step of HYMSY geometric processing chain (Figure 3) starts by pairing spectrometer and aerial images to the corresponding GPS-INS messages by matching the time stamps. Using the boresight calibration parameters, the orientations of the GPS-INS unit are converted to 
Northing-Easting-Altitude-Roll-Pitch-Yaw vectors associated with each spectrometer scan line and aerial image.

In traditional direct georeferencing processing these orientation data are then used directly with an external DEM. With high-resolution data this workflow will lead into large geometrical errors relative to pixel size because of two reasons. Firstly, using an external DEM makes it impossible to take small objects into account producing pixelwise georeferencing uncertainty relative to the altitude difference between the DEM and the real surface. With most airborne sensors this is not a problem as hyperspectral data pixel sizes are typically in the order of $1-10 \mathrm{~m}$, which is approximately in the same order as the DEM accuracy. With HYMSY the pixel size is typically $0.10-1.0 \mathrm{~m}$, increasing the accuracy requirement of the DEM/DSM by tenfold. Using a digital ground elevation model is not accurate enough for HYMSY data in most mapped areas, because it is necessary to take the vegetation and other raised surfaces into account. With these high resolutions, usage of an actual surface model is required instead of a terrain model. Secondly, the acquisition of an external DSM with a high enough co-registration accuracy with the spectrometer orientation data is bound to be laborious if not impossible. As a result, a high accuracy DSM that is co-registered accurately enough to spectrometer orientation data cannot be acquired externally but it must be generated within in the same processing chain.

The second step of the HYMSY geometric processing chain (Figure 3) adopts a photogrammetric method to generate the DSM from the aerial images collected simultaneously with the hyperspectral data. The photogrammetric software (PhotoScan Pro, v1.0.0, Agisoft) is fed with the aerial images and the image orientation data. The software uses a Structure-from-Motion algorithm [14] to find tie-points between the images and uses a block bundle adjustment to fit the observed points and camera positions together. This process produces a 3D surface model of the area and fine-adjusts the camera orientations above the model to match it accurately. Optionally, PhotoScan allows the introduction of ground control points (GCPs) measured with a RTK-GPS, which increases the global positioning accuracy of the model and camera positions to reach a precision in the order of a few centimeters. This is not a mandatory step as the internal accuracy between the 3D model and camera orientations is already high enough without the GCPs. The DSM and the camera orientations are an output from PhotoScan so they can be used in the next processing steps. As the final photogrammetric step, PhotoScan projects the aerial images over the DSM producing a high-resolution RGB orthomosaic (Figure 3).

As the onboard GPS-INS unit is a compact and economic one, it uses only a simple single band GPS receiver with an absolute spatial accuracy between 4-10 m. As a result, it is mandatory to increase the accuracy of the orientation data with the photogrammetric orientations of the aerial images. In the third step of the geometric processing (Figure 3), the photogrammetric camera orientations for the aerial image are converted to spectrometer orientations using the boresight calibration parameters. As the aerial images are taken only at $0.5 \mathrm{~Hz}$ rate while spectrometer scan lines are taken at $25 \mathrm{~Hz}$, interpolation is required to fuse the orientations. For this interpolation, the photogrammetric values are used directly and only the non-linear component of the high frequency GPS-INS data is added to the values.

In the fourth geometric processing step, the hyperspectral data are georectified using a traditional direct georeferencing algorithm (PARGE, 3.2beta, ReSe Applications Schläpfer, [12]). For PARGE, the following input is required: the radiometrically processed datacube, the fused spectrometer 
orientations, the photogrammetric DSM, the spectrometer geometric model, and the settings about the resolution and map extent. PARGE then projects the hyperspectral pixels over the DSM and produces a rasterized georeferenced datacube.

Figure 3. Flowchart of the HYMSY geometric processing chain. The radiometric (reflectance factor) processing (Section 3.1) is done prior to geometric processing. The yellow cylinders are input data, white cylinders are temporary data, and green cylinders are final output data products. Orange boxes are processing steps.

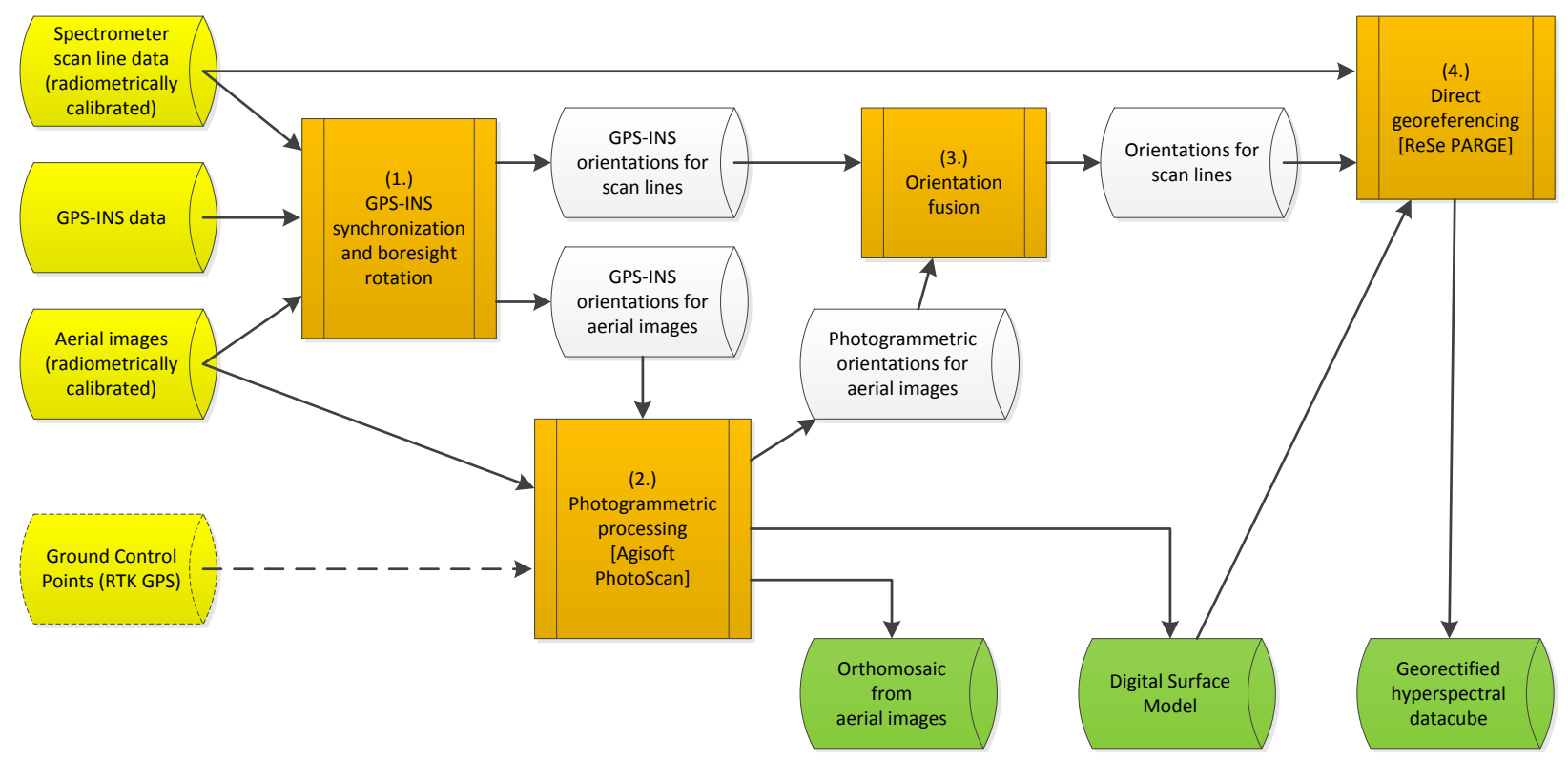

\section{Experimental Case Study}

\subsection{Signal to Noise Ratio}

The signal-to-noise ratio of the sensor was estimated by evaluating static measurements over a white reference panel (Labsphere Inc). Analysis was done on the binned 8-bit pixels. The minor changes in illumination intensity were removed from the data by dividing each spectrometer image with its average value. The pixelwise noise level was received by calculating standard deviation of each pixel intensity over time. The average pixelwise noise level was found to be 0.3849 counts. However the used method ignores possible variations in camera exposure time and gain. To evaluate this, differences between consecutive raw image average intensities were analyzed and a standard deviation of 0.2286-counts at full well was observed. By propagating the error from the pixelwise noise and the image-wide variation, the signal to noise ratio (SNR) for 16-bit images is estimated to be at least 518/1 at full well.

\subsection{Mapping Performance}

A major HYMSY campaign lasting throughout the summer of 2013 was performed on agricultural experimental fields of the CAH Vilentum University of Applied Sciences in Dronten, The Netherlands [15]. Objective of the campaign was to evaluate how UAV based hyperspectral 
observations could be adopted for mapping and monitoring of a variety of arable crops. On 16 July 2013 a mapping flight consisting of two individual flight lines was performed over an onion and winter wheat field (Figure 4). The flight altitude was programmed to the full legal maximum of $120 \mathrm{~m}$ and flight speed was $6 \mathrm{~m} / \mathrm{s}$ resulting in a Ground Sampling Distances (GSD) of $320 \mathrm{~mm}$ for the spectrometer data (cross-track) and $34 \mathrm{~mm}$ for the aerial images. To calibrate the data to reflectance factors, the $25 \%$ Spectralon panel was measured immediately before and after the flight.

In the July 2013 experiment the HYMSY was still fixed in a preliminary aluminum frame that tended to bend slightly between installations. Thus in this dataset the boresight calibration parameters were manually adjusted to overlap the aerial image and hyperspectral data. No other manual rubber sheeting was performed. Since March 2014, HYMSY is installed on a sturdy carbon fiber frame (Figure 1) removing the need for flight-wise manual boresight adjustments.

In absence of RTK-GPS ground control points, the global georeferencing accuracy of the dataset is limited to the $4-10 \mathrm{~m}$ accuracy of the single band GPS receiver in the GPS-INS unit. As the Figure 4 shows the internal georeferencing, accuracy of the datasets is much higher and, even with the old unstable frame, is in the order of a few spectrometer ground pixels $(0.15 \%$ pixel $)$.

Figure 4. A collage image of the HYMSY dataset from a single flight at $120 \mathrm{~m}$ altitude. A RGB orthomosaic at $34 \mathrm{~mm}$ GSD generated from the aerial images (Left background). The Digital Surface Model at $77 \mathrm{~mm}$ resolution visualized with hillshading effect (Right background). A false color composite ( $\mathrm{RGB}=800,650,550 \mathrm{~nm}$ respectively) of the hyperspectral dataset of the first flight line at $320 \mathrm{~mm}$ GSD (Front). The circles mark the locations of spectra displayed in Figure 5.

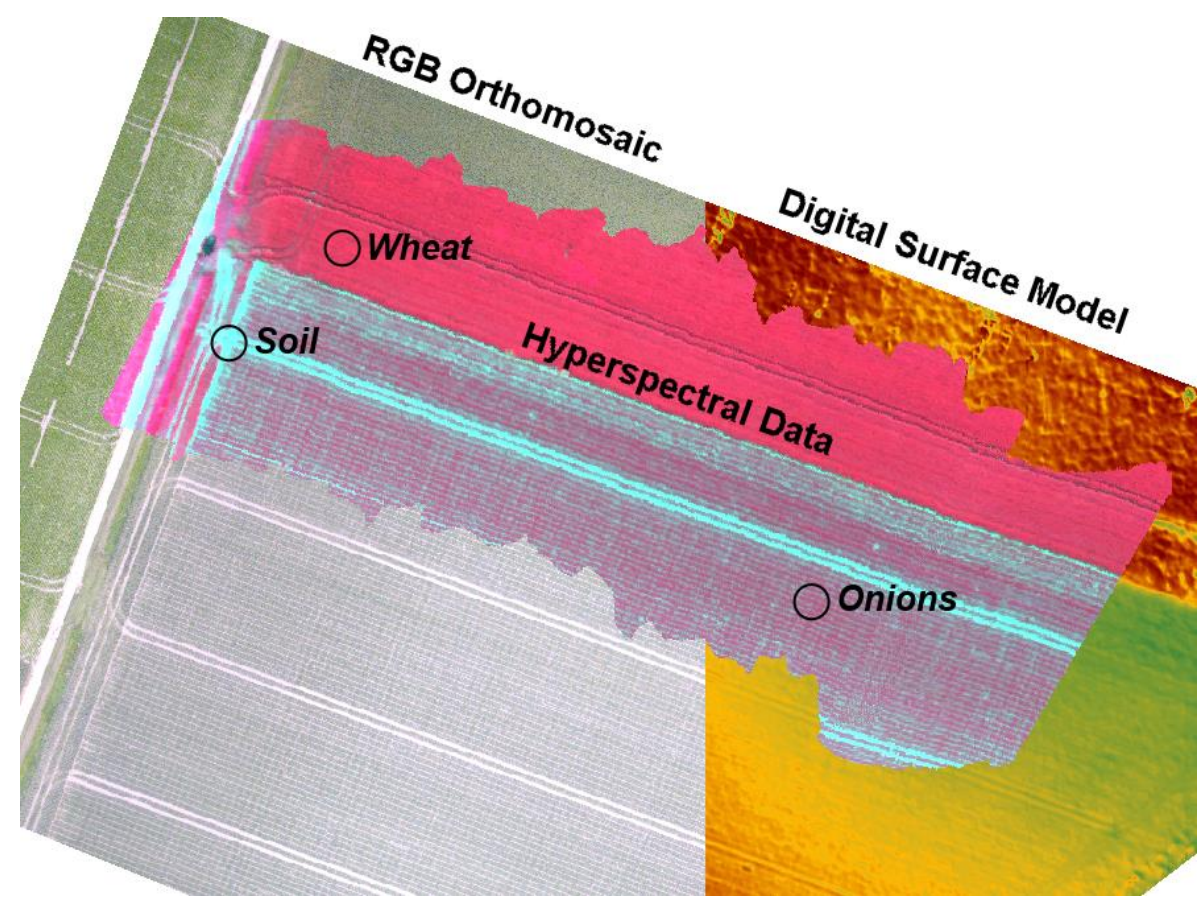

After the flight, field spectra were collected of the crops and soil using an ASD FieldSpec HandHeld 2 spectrometer. The HYMSY spectra were selected from the image by averaging the spectra corresponding to the approximate location of the field measurements. The comparison of the field and airborne spectra is shown in Figure 5. In general the spectra match well, but also some differences are 
detectable. The onion spectra differ because the field spectra were collected over the crop rows only, while airborne data was averaged also over the paths. For this reason, the airborne onion spectra show a higher contribution of bare soil, increasing the reflectance in the visible part of the spectrum and lowering it in the near-infrared part. For the soil spectra the effect is opposite as tractor tracks were not wide enough to collect airborne spectra completely without the influence of spectral mixing of vegetation. The wheat field had a homogenous and continuous vegetation cover, which results in a good correspondence of the airborne and field spectra.

In this dataset, HYMSY systematically overestimates the reflectance factor in blue. This was caused by a poorly performed calibration procedure with the Spectralon panel, where the UAV and the person holding it were blocking part of the diffuse light. This error source can be minimized during the calibration by holding the UAV as high as possible and standing as far as possible from the Spectralon panel. The spectra also still show some noise-like vibrations but in the central wavelength region of the spectra these are mostly caused by uncertainties in the first laboratory calibration parameters of the system. The accuracy was improved in recalibration but due to compatibility issues this can only be applied for later datasets.

Figure 5. Comparison of HYMSY spectra and ground reference (ASD FieldSpec HandHeld 2) spectra. The HYMSY spectra were picked from the datacube shown in Figure 4 by averaging pixels over a small area (10-100 pixels) close to the estimated location of the ground reference spectra. The sampled areas do not match perfectly and thus some deviation is expected especially with the soil and onion samples. Especially in the wheat spectra in this dataset HYMSY overestimates reflectance factors in the blue region due to poorly performed Spectralon calibration phase where part of the diffuse light got blocked by the person holding the UAV.

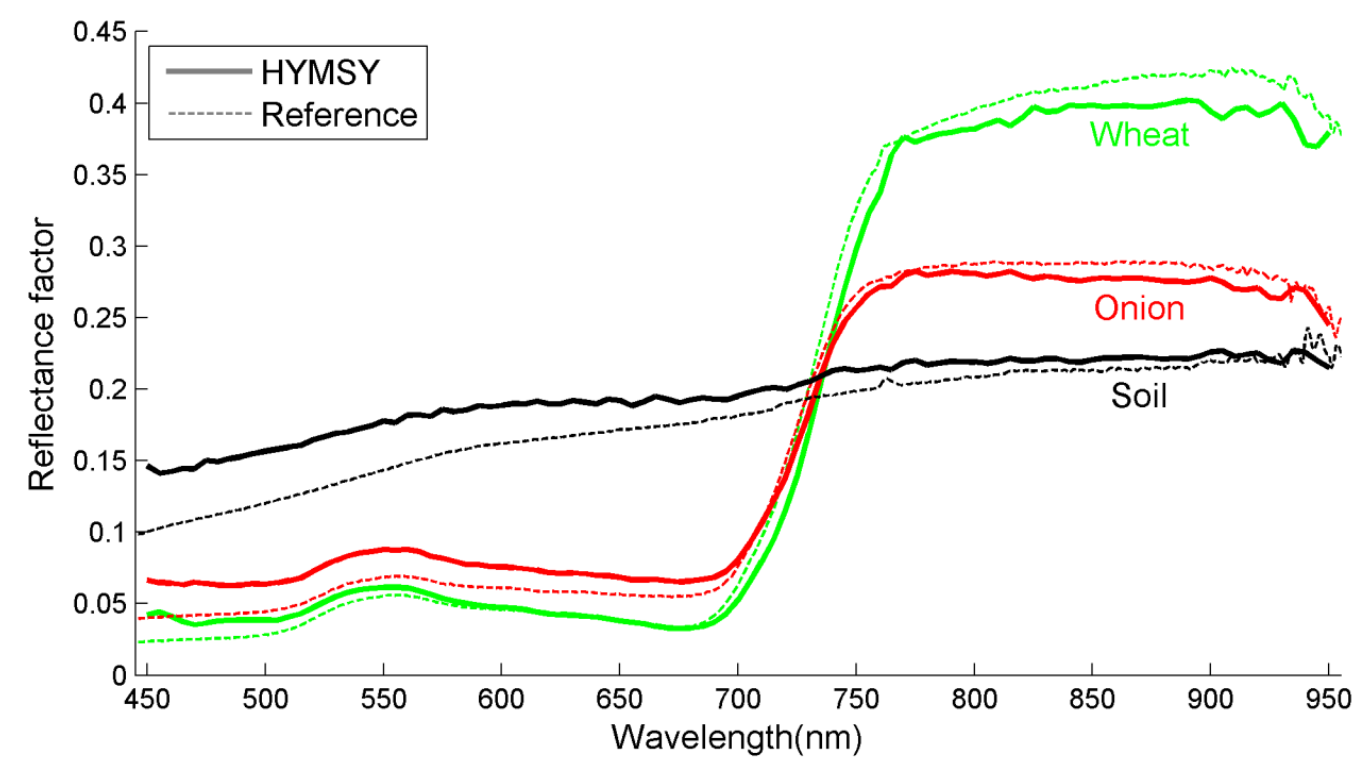

\section{Discussion}

In general, the design goals of the HYMSY have been reached. During 2013 and 2014 the HYMSY performed more than 200 octocopter flights at more than 70 sites. On a typical flight, we mapped a 
2-10 ha area producing a RGB orthomosaic at $1-5 \mathrm{~cm}$ resolution, a DSM in 5-10 $\mathrm{cm}$ resolution, and a hyperspectral datacube at $10-50 \mathrm{~cm}$ resolution. Vegetated targets included potatoes [16], wheat, sugar beets, onions, tulips, agricultural soils, heathlands, coral reefs [17], and tropical forests. The low weight and size of the whole UAV based mapping system has given us the flexibility to easily perform all these mapping tasks. We also found the added value of the high-resolution photogrammetric camera to be high in multiple aspects. With support of photogrammetric processing, the small GPS-INS unit has proven to be accurate enough for georectification. The co-registered RGB orthomosaic gave a visual identification support of objects in the coarser hyperspectral maps and provides a recognizable result for non-professional audiences. In preliminary tests, we have also found the vegetation height maps generated from DSMs to be useful in image classification tasks.

Although the HYMSY is a fully operational instrument whose design is considered successful for vegetation mapping and monitoring applications, the authors recommend some improvements in the design before cloning the system. The HYMSY was originally designed around the PhotonFocus SM2 industrial camera because it also housed a Digital Signal Processor (DSP) and microSD slot in one package, allowing standalone operation without external data storage. Unfortunately, the performance of SM2 did not fully match our expectations. Firstly, the DSP's driver architecture prevented writing of data to the SD-card while acquiring new imagery, which decreased the maximum frame rate of the SD storage system to unacceptably low frequencies. As an alternative, the external Raspberry PI was added as a data sink with approximately $100 \mathrm{~g}$ of extra weight. Secondly, the DSP was found to be slightly underpowered to the task. Although the SM2 is theoretically capable of reaching frame rates of up to $160 \mathrm{fps}$, our application, including binning, triggering, GPS-INS communication, and Ethernet output, reached a maximum stable frame rate of only $25 \mathrm{fps}$ in 8-bit output mode. As the camera sensor is typically run with only 12 -ms exposures, a system could be operated with two to three times faster frame rate by using faster processing electronics. For these reasons it is recommended to design a future system around either a more powerful DSP or a simpler binning-enabled GigE-camera and an external board-computer.

Because of the above-mentioned issues, the frame rate of the current HYMSY can be considered to be low and in many cases this limits the usability of the system for ultra-high-resolution mapping. Fixed wing UAVs and aircrafts have a minimum airspeed to prevent stalling. With UAV platforms this is usually within a range of $10-15 \mathrm{~m} / \mathrm{s}$. Although rotary wing UAVs do not have a minimum airspeed in stalling aspect, for our octocopter platform the slowest smooth flight speed is around $2 \mathrm{~m} / \mathrm{s}$. At slower speeds, the UAV autopilot progresses by "hopping forward" with more tilting movements than is desirable for pushbroom mapping. The sensor frame rate and the minimum flight speed define the along track Ground Sampling Distance. Since our octocopter has a minimum speed of $2 \mathrm{~m} / \mathrm{s}$ and we use a frame rate of $25 \mathrm{fps}$, the best possible GSD and output resolution is on the order of $10 \mathrm{~cm}$. For a fixed wing UAV the best possible GSD at $25 \mathrm{fps}$ would only be on the order of $40-60 \mathrm{~cm}$. For a future system, effort should be placed on increasing the frame rate.

To increase the frame rate, the spectrometer currently runs in 8-bit mode, which decreases the radiometric quality of the spectrometer output. In an ideal case, the raw pixels could be read in 12-bit resolution, and pixels binned with a $4 \times 4$ window could contain the full 16-bits of data. Based on the manufacturers specifications, the SNR of such a binned pixel at full well should be up to 1200/1 (10.2 bits). Unfortunately, this high performance is not currently achieved as the pixels are read in and 
output as 8-bit numbers, decreasing the numerical resolution of a single pixel to 256/1. For the georectified dataset however, the radiometric resolution on full-well is expected to be slightly higher because in the course of georectification, the raw pixels are merged with neighboring pixels both in the spectral and spatial domain which slightly improves the SNR. Since increasing the radiometric resolution would be very beneficial, one of our short-term goals is to investigate if optimization of the DSP program would allow us to increase the numerical resolution of binning and output to 12-16 bits without significantly compromising the frame rate.

In the case of the octocopter, radiometric calibration using reflectance reference panels was found to be a practical method. However, if the processing chain is used for longer flight times or larger distances from the takeoff spot, which is the situation for most fixed wing systems, another radiometric calibration method should be considered [13]. Using the photogrammetric methods in geometric processing does not restrict the possibilities for radiometric calibration method and the use of atmospheric transmission models or onboard incident light sensors can thus be explored.

The photogrammetric processing chain has clear advantages over the traditional direct georeferencing that is used by most, if not all, current commercial pushbroom systems: (1) The method allows exploitation of a cheaper and more lightweight GPS-INS system, (2) It is capable of projecting pushbroom data over an up-to-date DSM with fine details of even temporary targets, such as parked cars, vegetation, etc. (3) It allows fully automated georectification with an internal accuracy of a few pixels and a global accuracy depending on the onboard GPS/GNSS receiver.

The drawbacks of the photogrammetric method include uncertainty in success of the photogrammetric processing. Lack of detectable features in the imaged landscape may result in complete failure of the photogrammetric processing. The photogrammetry also will fail if the overlap of aerial images is too low or when the image parallax is too large compared to the complexity of the target. For example, we experienced failure over a forest without leaves when flying too fast. Such issues can be addressed by increasing the overlap between subsequent images, which can be achieved by decreasing flight speed or increasing the frame rate of the aerial image camera, if possible. Failure or severe distortions are also likely to occur above clear water bodies where the water surface refracts underwater objects in the image or waves prevent detection of any stable features.

The photogrammetric processing also introduces some requirements for the flight path of the mapping. In general, the aerial image dataset should consist of images with a two-dimensional spatial distribution significantly larger than the accuracy of the GPS receiver. While the photogrammetric process probably still succeeds in image alignment when the spatial separation of extreme images is smaller than the GPS accuracy, the 3D model and camera view angles become tilted or even rotated completely upside-down. In our experience, this can also happen with a long (>100 m) flight consisting only of a single linear path. One way to solve this is by adding ground control points measured with an RTK-GPS to the photogrammetric processing, unfortunately adding a manual step to the processing chain. This is usually not necessary and a fully automated processing can be performed if the flight lines are planned such that this aspect is taken into account. Simply adding a 10-20 m L-branch to the beginning or end of the flight line, or by flying multiple parallel flight lines with some side-overlap is enough to stabilize the rotation.

Currently the only manual, but optional, step in the georectification process is the selection of the RTK-GPS ground control points on the aerial images. The RTK GPS points are needed to improve the 
global positioning accuracy above the single band GPS accuracy of the GPS-INS unit. Adding a miniaturized RTK GPS receiver to the imaging system could potentially improve the georectification accuracy to the level of hyperspectral pixel size where other ground control points and manual processing steps would not be needed at all. Even in this scenario however, introduction of additional GCPs is expected to be beneficial for geometric accuracy.

The photogrammetric processing as presented in this paper is a powerful replacement for the high accuracy GPS-INS systems and allows the introduction of new approaches to the technical design of UAV mapping systems. As presented in this study, the technical requirements of adding a photogrammetric capability to mapping system are minor. The system requires a photogrammetric camera, which is synchronized to the pushbroom acquisition. The single requirement of the camera is that its pixel size on the ground should roughly be at least on the same order of magnitude as the required alignment accuracy. Thus, the introduction of photogrammetric processing allows for a decrease in the quality and size of the inertial measurement unit.

The image data collected by the HYMSY have the potential to allow for the characterization of agricultural crop variation within a parcel at specific observation points during the growing season (Figure 5). First results have shown the application of HYMSY for a crop monitoring experiment in potato [16]. Vegetation indices based on red-edge bands were evaluated on their relation with measured chlorophyll concentrations in the field. The vegetation indices showed a good linear relation with relevant crop traits and the empirical model based on the chlorophyll red-edge index was used to derive continuous chlorophyll maps using the acquired hyperspectral data cubes. Follow up research will focus on the automated detection of soil, vegetation and shadow components within the image up to the level of individual plant detection by using image analysis techniques like super resolution mapping [18]. At the individual plant level, crop properties can be estimated and transformed to task maps for variable-rate application within the agricultural parcel.

\section{Conclusions}

We have developed a lightweight hyperspectral mapping system (HYMSY) specifically designed for rotor-based UAV and presented the potential for agricultural mapping and monitoring applications. The system has been mostly constructed from off-the-shelf components, with only the synchronization electronics and the frame being custom-made. At ready-to-fly configuration, the system weighs only $2.0 \mathrm{~kg}$, allowing for mounting on smaller UAVs than is possible with other commercially available spectrometers. The HYMSY combines a custom-made pushbroom spectrometer (400-950 nm, $9 \mathrm{~nm}$ FWHM, 25 lines/s, 328 px/line) with a photogrammetric camera (16 MPix images every $2 \mathrm{~s}$ ), which are synchronized to a miniature GPS-Inertial Navigation System.

An important improvement has been developed in the processing chain for georectification of the hyperspectral pushbroom data. The processing chain uses a photogrammetric algorithm to produce a Digital Surface Model (DSM) and provides high accuracy orientation of the system over the DSM. Producing an up-to-date DSM is part of the processing chain, so no external data are required, in contrast to traditional direct georeferencing approaches. The processing chain is capable of georectifying pushbroom data fully automatically, with an internal accuracy of a few pixels. Because of photogrammetric processing, the internal georeferencing exceeds the accuracy of the low 
performance GPS-INS unit. However, implementation of the proposed approach introduces new requirements for the flight path planning of the mapping to secure enough overlap of aerial images and includes flying multiple parallel flight lines with some side-overlap to stabilize the rotation of the DSM. Because of the mentioned advantages, the authors recommend adding such photogrammetric methods to processing chains off all high-resolution pushbroom systems including ones mounted on traditional manned airborne systems.

\section{Acknowledgments}

This project was funded by Interreg project Smart Inspectors (Project number: I-1-03=176) and performed under WageningenUR Unmanned Aerial Remote Sensing Facility (http://www.wageningenur.nl/uarsf).

\section{Author Contributions}

Juha Suomalainen is the main designer and constructor of the HYMSY and the processing chain; Niels Anders provided expertise in photogrammetric processing; Shahzad Iqbal and Dirk Hünniger designed and programmed the spectrometer acquisition software; Gerbert Roerink provided and processed the ground reference data; Jappe Franke, Philip Wenting, and Harm Bartholomeus took part and supported in development and measurements; and Lammert Kooistra and Rolf Becker supervised the research and gave their valuable insight to the project.

\section{Conflicts of Interest}

The authors declare no conflict of interest.

\section{References}

1. Schaepman, M.E.; Ustin, S.L.; Plaza, A.J.; Painter, T.H.; Verrelst, J.; Liang, S. Earth system science related imaging spectroscopy_An assessment. Remote Sens. Environ. 2009, 113, S123-S137.

2. Clevers, J.G.P.W.; Kooistra, L. Using hyperspectral remote sensing data for retrieving canopy chlorophyll and nitrogen content. IEEE J. Sel. Top. Appl. Earth Obs. Remote Sens. 2012, 5, 574-583.

3. Clevers, J.G.P.W.; Kooistra, L.; Schaepman, M.E. Estimating canopy water content using hyperspectral remote sensing data. Int. J. Appl. Earth Obs. 2010, 12, 119-125.

4. Colomina, I.; Molina, P. Unmanned aerial systems for photogrammetry and remote sensing: A review. ISPRS J. Photogramm. Remote Sens. 2014, 92, 79-97.

5. Van Blyenburgh, P. 2014 RPAS Yearbook-RPAS: The Global Perspective, 12 ed.; Blyenburgh \& Co: Paris, France, 2014.

6. Laliberte, A.S.; Winters, C.; Rango, A. UAS remote sensing missions for rangeland applications. Geocarto Int. 2011, 26, 141-156.

7. Turner, D.; Lucieer, A.; Watson, C. An automated technique for generating georectified mosaics from ultra-high resolution unmanned aerial vehicle (UAV) imagery, based on structure from motion (SfM) point clouds. Remote Sens. 2012, 4, 1392-1410. 
8. Jaakkola, A.; Hyyppä, J.; Kukko, A.; Yu, X.; Kaartinen, H.; Lehtomäki, M.; Lin, Y. A low-cost multi-sensoral mobile mapping system and its feasibility for tree measurements. ISPRS J. Photogramm. Remote Sens. 2010, 65, 514-522.

9. Zarco-Tejada, P.J.; González-Dugo, V.; Berni, J.A.J. Fluorescence, temperature and narrow-band indices acquired from a UAV platform for water stress detection using a micro-hyperspectral imager and a thermal camera. Remote Sens. Environ. 2012, 117, 322-337.

10. Hruska, R.; Mitchell, J.; Anderson, M.; Glenn, N.F. Radiometric and geometric analysis of hyperspectral imagery acquired from an unmanned aerial vehicle. Remote Sens. 2012, 4, 2736-2752.

11. Lucieer, A.; Malenovský, Z.; Veness, T.; Wallace, L. HyperUAS-Imaging spectroscopy from a multirotor unmanned aircraft system. J. Field Robotics 2014, 31, 571-590.

12. Schläpfer, D.; Richter, R. Geo-atmospheric processing of airborne imaging spectrometry data. Part 1: Parametric orthorectification. Int. J. Remote Sens. 2002, 23, 2609-2630.

13. Van Duijvenbode, J. Incident Light Monitoring for UAV-Acquired Image Correlation. Master's Thesis, Wageningen University, Wageningen, The Netherlands, 2014.

14. Jebara, T.; Azarbayejani, A.; Pentland, A. 3D structure from 2D motion. IEEE Signal Process. Mag. 1999, 16, 66-84.

15. Mucher, S.; Roerink, G.; Franke, J.; Suomalainen, J.; Kooistra, L. Monitoring agricultural crop growth: comparison of high spatial-temporal satellite imagery versus UAV-based imaging spectrometer time series measurements. In Proceedings of 2014 EGU General Assembly, Vienna, Austria, 27 April-2 May 2014; p. 15788.

16. Kooistra, L.; Suomalainen, J.; Iqbal, S.; Franke, J.; Wenting, P.; Bartholomeus, H.; Mücher, S.; Becker, R. Crop monitoring using a light-weight hyperspectral mapping system for unmanned aerial vehicles: Firs results for the 2013 season. In Proceedings of 2013 Workshop on UAV-based Remote Sensing Methods for Monitoring Vegetation, Cologne, Germany, 9-10 September 2013; pp. 51-58.

17. Suomalainen, J.; Mucher, S.; Kooistra, L.; Meesters, E. Mapping health of Bonaire Coral Reefs using a lightweight hyperspectral mapping system-First results. In Proceedings of 2014 EGU General Assembly, Vienna, Austria, 27 April-2 May 2014; p. 15619.

18. Tolpekin, V.A.; Stein, A. Quantification of the effects of land-cover-class spectral separability on the accuracy of Markov-random-field-based superresolution mapping. IEEE Trans. Geosci. Remote Sens. 2009, 47, 3283-3297.

(C) 2014 by the authors; licensee MDPI, Basel, Switzerland. This article is an open access article distributed under the terms and conditions of the Creative Commons Attribution license (http://creativecommons.org/licenses/by/4.0/). 